\title{
Liquid micro pulsed plasma thruster
}

\author{
Agnieszka Szelecka, \\ Jacek Kurzyna, \\ Dariusz Daniłko, \\ Serge Barral
}

\begin{abstract}
A new type of pulsed plasma thruster (PPT) for small satellite propulsion is investigated, of which the most innovative aspect is the use of a non-volatile liquid propellant. The thruster is based on an open capillary design. The thruster achieved a thrust-to-power ratio above $45 \mu \mathrm{N} / \mathrm{W}$, which constitutes a 5-fold improvement over the water-propelled pulsed plasma thruster, and which is also slightly above the performance of a similarly sized PPT with a solid propellant.
\end{abstract}

Key words: electric propulsion $\bullet$ pulsed plasma thruster $\bullet$ liquid propellant

A. Szelecka ${ }^{\bowtie}$, J. Kurzyna, D. Daniłko Institute of Plasma Physics and Laser Microfusion (IPPLM),

23 Hery Str., 01-497 Warsaw, Poland,

Tel.: +48 22638 1460, Fax: +48 226668372 ,

E-mail: agnieszka.szelecka@ifpilm.pl

S. Barral

QuinteScience SERGE BARRAL,

31/33 Rozbrat Str., 00-429 Warsaw, Poland

Received: 27 November 2014

Accepted: 24 March 2015

\section{Introduction}

Pulsed plasma thrusters seem to be well-suited for nano-satellites (CubeSats), because of their small size, reliability, high specific impulse, low power consumption and the accuracy of orbital maneuvers. Plasma propulsion devices may be roughly divided into three categories based on the phase of the propellant used: solid, liquid or gas. Each of the propellant types has both its merits as well as disadvantages. The choice of the propellant is usually a first stage in the development of a thruster as the design of the whole device is often centered around the type of the propellant to be used.

Solid propellants that have been used for electric propulsion include: powdered propellants, composite propellants as well as sintered, seeded or impregnated teflon and fluorocarbons. Solid propellants have many benefits: there is no need for tanks, valves and feed lines, which leads to the improved reliability through the simplicity of the system. However, PPTs that use solid propellant offer little control over the amount of the propellant that is used to generate thrust in individual pulses. This results in low repeatability of the impulses. Another concern associated with using solids for pulsed propulsion systems is that the mass of the propellant that may potentially be lost due to late-time ablation [1] may be large.

As far as gaseous propellants are concerned, argon, nitrogen, xenon and water vapor have been tested [2]. The main benefits of using gaseous propellants are the performance repeatability and the 
elimination of late-time ablation [2]. Among the main drawbacks of using gas propellants in PPTs is the increased complexity of the feeding system as well as the need to provide precise synchronization between the gas feeding system and the igniter. Failing to achieve good coupling between the two subsystems may be a possible source of large mass losses.

It seems that the need for a precise synchronization could be avoided by using a liquid propellant instead of a gas propellant. However, when it comes to liquid propellants, the biggest obstacle for using them in pulsed thrusters seems to be the rapid vaporization of most liquids at low pressures. This is especially true for water, which has been thoroughly investigated as a potential PPT propellant, mainly because of its low price and the ease of handing [2]. For example, in a study conducted by Ziemer [3], water was introduced in a vapor state through a gas valve, generating a train of discharge pulses with a very high repetition rate so as to increase the utilization rate of the propellant introduced during one valve opening. The water PPT concept was pursued by Kakami and Koizumi between 2003 and 2005 [4, 5] with a drop-on-demand propellant feeding system based on an electromechanical injector. Another drawback of using water as a propellant is its expansion upon freezing, which impedes storage and feeding in orbital conditions $[4,5]$.

In order to develop a liquid PPT that takes advantage of the increased repeatability as compared to solid PPTs an alternative propellant is therefore required, which is expected to fulfill the following requirements: an excellent chemical stability, a liquid phase stability over a wide range of temperatures and pressures, dielectric properties, a high storage density, a low evaporation rate in vacuum and lack of hazard to organic life. After careful evaluation of several candidates perfluoropolyether (PFPE) [1] was chosen for this study.

\section{Thruster design}

The general concept of a pulsed plasma thruster is as follows. The propellant is positioned between the two electrodes connected to high voltage power source. The electrodes constitute the acceleration channel. When voltage is applied to the electrodes, the initiating discharge is possible in place of minimum inductance near the propellant surface. Discharge causes ablation and ionization of a tiny amount of the propellant. Transforming the propellant into a plasma allows for its acceleration through the Lorenz force $\vec{F}$. This force is created by the discharge current $\vec{j}$ which interacts with self-induced magnetic field $\vec{B}$. This is the general mechanism that the concept of a liquid PPT was based on [6]. The electrodes and the formation of the thrust are presented in Fig. 1.

Since it was only meant as a proof of concept, the first prototype of the thruster that was investigated used a very small tank capable of storing only $50 \mu \mathrm{l}$ of the propellant. The general layout of the thruster

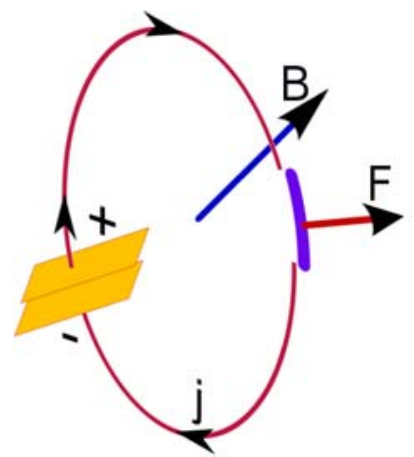

Fig. 1. The principle of thrust generation: the interaction between the discharge current $\vec{j}$ and the self-induced magnetic field $\vec{B}$.

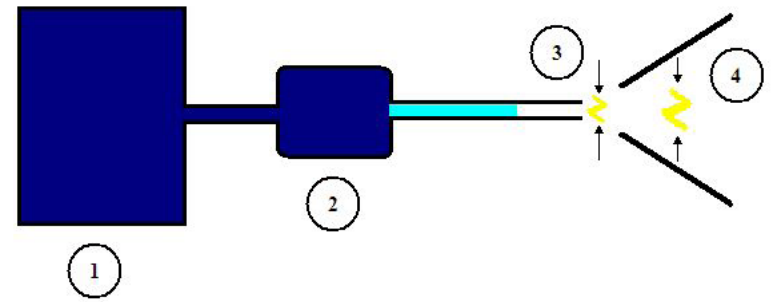

Fig. 2. Conceptual design of the liquid micro pulsed plasma thruster with open capillary. Item 1: tank; item 2: syringe pump; items 3 and 4: ignition and main discharge electrodes [5].

is shown in Fig. 2, with the tank indicated as the item 1 . The micro-pump, indicated as the item 2 , uses a miniaturized step motor coupled to a lead screw that directly drives a $2 \mathrm{~mm}$ diameter piston. This allows for a precise control of the propellant volume being injected. Additionally, a temperature sensor located near the end of the capillary enables an on-board micro-controller to monitor the propellant temperature and compensate for its thermal expansion inside the tank by adjusting the position of the piston. The item 3 shows the spark gap, which runs across the $0.8 \mathrm{~mm}$ diameter orifice of the capillary. This ignition spark gap is responsible for triggering the main discharge [3].

The anticipated ablation process is illustrated in Fig. 3. The propellant moves into the open capillary and is subsequently ablated by the spark gap. The generated ionized vapor triggers the main discharge which accelerates ablated plasma [3]. In this first liquid micro pulsed plasma thruster prototype, a single step of the motor driving the syringe pump corresponds to the delivery of $31 \mathrm{nl}$ of the propellant (the corresponding mass depends on the temperature but is approximately equal to $50 \mu \mathrm{g}$ ) [3].

Tests were carried out with nominal propellant mass bit around $5 \mu \mathrm{g}$ - which is equivalent to $3 \mathrm{nl}$ - of the chosen perfluoropolyether; the radius of propellant drop is about $0.1 \mathrm{~mm}$. In Fig. 3 an arrow depicts the small discharge between the two small electrodes. Dark grey rectangles represent the subsequent doses of a the propellant.

The photo of a liquid micro pulsed plasma thruster is presented in Fig. 4. It consists of the following parts: the main electrodes, which are on the front of the thruster (1), the capacitor bank (2), which consists of 20 ceramic capacitors storing energy at the level of $1 \mathrm{~J}$, a small step motor (3) and electronics 


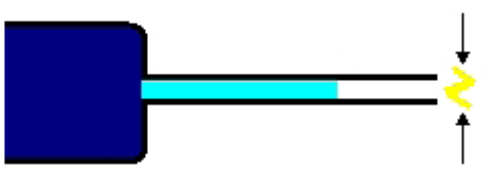

b)

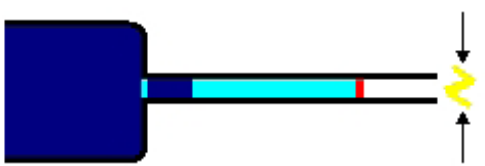

c)

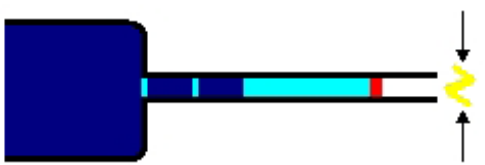

d)

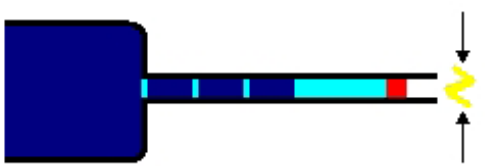

e)

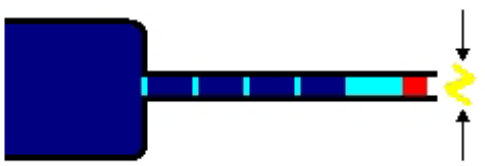

Fig. 3. An illustration of subsequent stages of the foreseen ablation process. Grey color inside capillary represents the chosen propellant. Dark grey color elements inside the capillary denote further dosage in accordance with the numbering from a) to e). At the end of the capillary is the volume of the ablated propellant, and outside of the capillary there is a small discharge [3].

constructed in Spain by Najera Aerospace (4) which controls the piston [1].

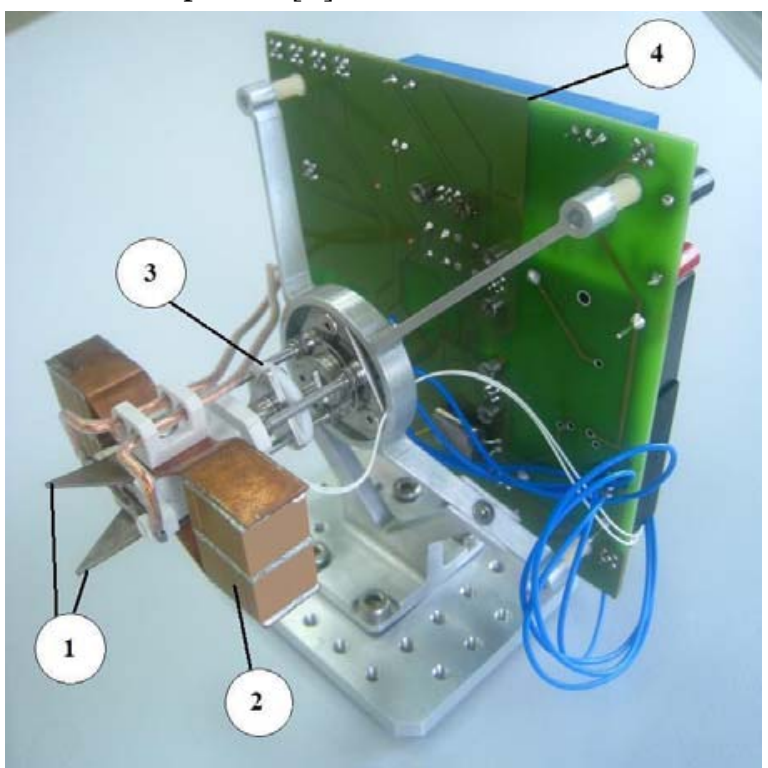

Fig. 4. The assembly of a thruster consisting of the two main electrodes (1), the ceramic capacitor bank (2), the step motor (3) and electronics (4) [1].

\section{Experimental setup}

\section{The ablation test setup}

Figure 5 presents an auxiliary device that was used to examine the mass flow rate of the propellant during the igniting discharge. The propellant is fed to the area between the electrodes with the use of capillary forces. The whole system was weighed before and after performing a few hundred discharges in order to calculate the average mass bit per ignition.

\section{The thrust measurements}

In order to measure impulses generated by the thruster, a very precise thrust stand was designed and manufactured by a Swiss company Mecartex, capable of measuring impulses in the $\mu$ Ns range. A photo of the thrust balance inside the vacuum chamber in the PlaNS laboratory at the Institute of Plasma Physics and Laser Microfusion in Warsaw is presented in Fig. 6. The stand works in the horizontal plane and is provided with angular sensors, adjustable plane and a regulating device.

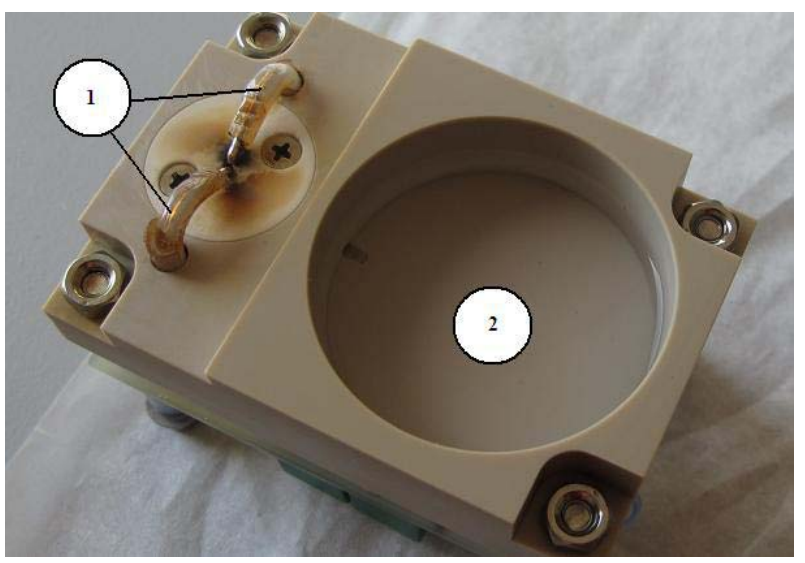

Fig. 5. The capillary used for testing the ablation process. It consisted of electrodes (1) - counterparts of the ignition electrodes in liquid micro pulsed plasma thruster - and a tank with the propellant (2).

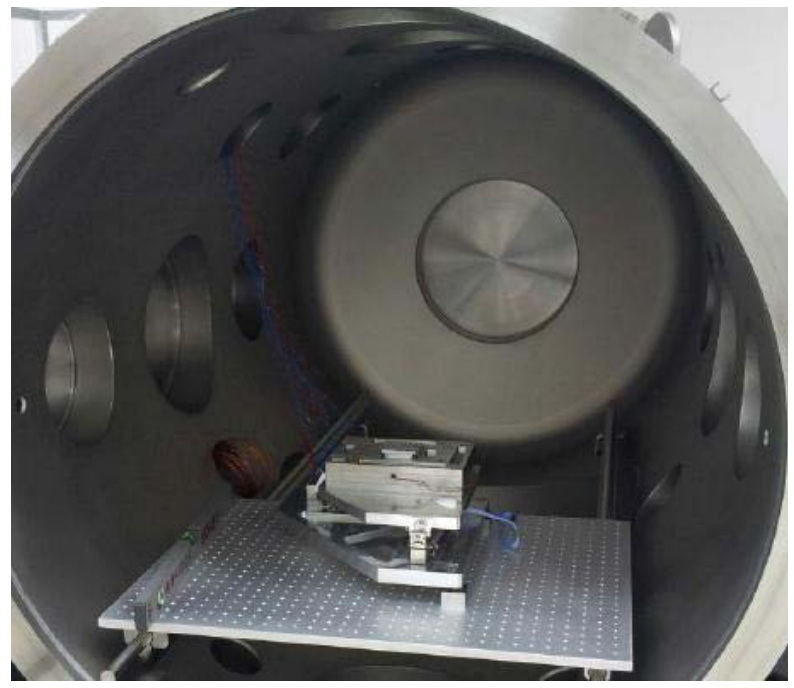

Fig. 6. Photograph of the thrust balance inside the vacuum chamber in the PlaNS laboratory. 
The pulses generated by the PPT trigger oscillations of the thrust stand. By measuring the amplitude and the frequency of the oscillations, it is possible to calculate the impulse bit:

$$
I=m \omega A=\sqrt{k m A}
$$

Here $A$ is the amplitude of the oscillations, $\omega$ is the angular frequency and $m$ is the constant moving mass. In order to calculate the impulse bit, both the stiffness $k$ and the nominal frequency must be known. Frequency is determined with precision of the order of $1 \%$ [1]. The derivation of Eq. (1) is presented in Appendix.

\section{Experimental results}

\section{Results of the ablation test}

With the use of the capillary shown in Fig. 5 it was established that the anticipated principle of operation of the thruster, according to which the spark ignition ablates the propellant for the main discharge to accelerate it, is not valid. The results of the experiment suggest that most of the ablated products are actually generated by the main discharge.

\section{The thrust measurements}

During the test campaign, more than a few thousand discharges were performed. The spark electrodes were powered by an ignition transformer with primary voltage set to around $300 \mathrm{~V}$, giving about $20 \mathrm{kV}$ in
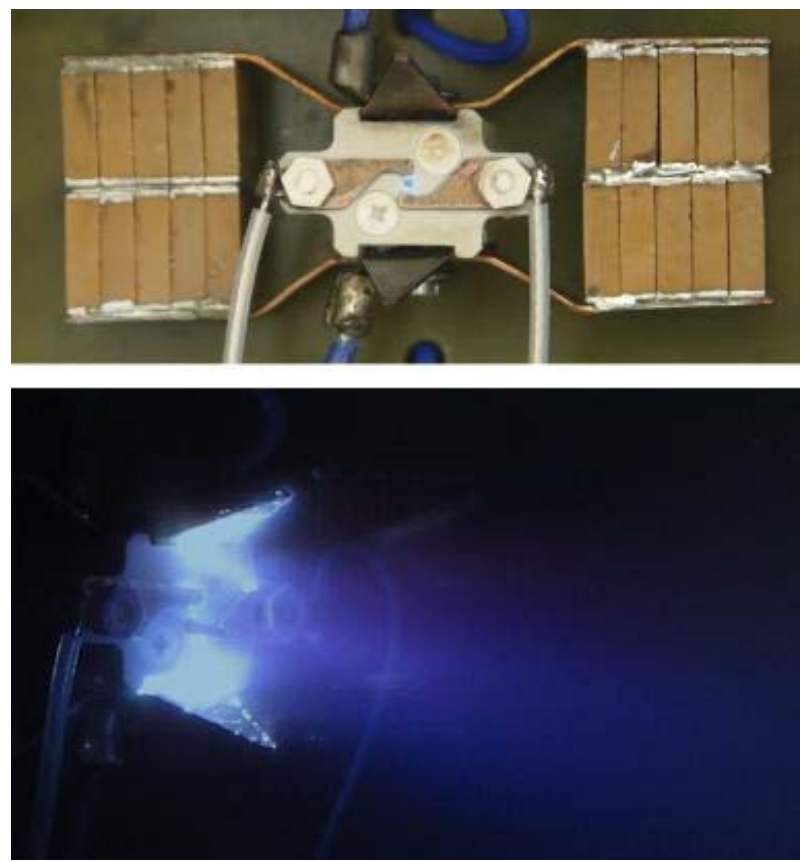

Fig. 7. On the top, a photograph of the thruster with a small discharge is presented. The light blue color between the small electrodes indicates the path of the discharge that ionizes the propellant. At the bottom, the main discharge at $900 \mathrm{~V}$ is shown, which is responsible for the acceleration and thrust generation.

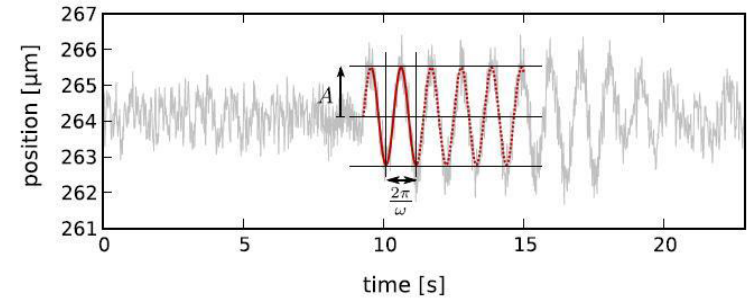

Fig. 8. Filtered signal with a fitting sinusoid (red) [1].

the secondary. The photo of the spark ignition is presented in Fig. 7 (top). The main discharge was triggered by a voltage applied to the main electrodes of the thruster. The voltage of the discharge was set to $750 \mathrm{~V}$ in the first batch of tests and was increased to $900 \mathrm{~V}$ in the second batch. At the bottom of Fig. 7 a photograph of the discharge at $900 \mathrm{~V}$ can be seen. The background pressure during the experiments was in the $10^{-6}$ mbar range.

An example of the raw signal obtained with the use of a thrust stand is presented in Fig. 8, along with a filtered signal and a sinusoid (red) that fits the data [1]. In order to calculate the impulse bit of Eq. (1), the amplitude and frequency have to be extracted from the signal shown in Fig. 8. The sine fitting makes the calculation of frequency straightforward without the need to use a Fourier transform. The first four periods were used for fitting, however, the results were roughly independent of the number of extrema that was used [1]. The procedure was conducted several times to obtain the average value of the impulse [1]. The average impulse bit was estimated to be $25 \mu \mathrm{Ns}$ at $700 \mathrm{~V}$ and $45 \mu \mathrm{Ns}$ at $1000 \mathrm{~V}$ [1].

\section{Conclusions}

In this article an experimental characterization of PPT operation with perfluoropolyether was shown, performed in the PlaNS laboratory at the Institute of Plasma Physics and Laser Microfusion in Warsaw.

The experiments proved that a pulsed plasma thruster using liquid PFPE as a propellant is a valid concept that may challenge the existing PPTs that use solid propellants. The liquid PFPE is free from most of the shortcomings of water and other liquids that were used as a PPT propellant in the past.

The thrust-to-power ratio obtained with the liquid micro pulsed plasma thruster reached $45 \mu \mathrm{Ns} / \mathrm{J}$, which proved to be a few times higher than that obtained with a PPT propelled with water [2] and slightly better than the thrust-to-power ratio obtained with a teflon PPT [7].

The results suggest that the role of the spark ignition in creating the ablated plasma is smaller than anticipated, which means that in order to provide a detailed description of the ablation mechanism, further studies are required.

Acknowledgments. The research leading to these results has received funding from the European Community's Seventh Framework Programme (FP7/2007- 
2013) under grant agreement no. 283279 for the L- $\mu$ PPT project. IPPLM has also received financial support from the Polish Fund for Science in years 2012-2014 for the execution of a partially funded international project.

\section{References}

1. Barral, S., Kurzyna, J., Szelecka, A., Rachubiński, H., Remírez, E., Martín, R., Ortiz, P., Alonso, J., Bottinelli, S., Mabillard, Y., Zaldívar, A., Rangsten, P., \& Koppel, C. (2014). First experimental characterization of a pulsed plasma thruster with non-volatile liquid propellant. In Proceedings of Space Propulsion Conference, 19-22 May 2014. Cologne, Germany.

2. Rezaeiha, A., \& Schönherr, T. (2013). Overview of alternative propellants for use in PPT. In 29th International Symposium on Space Technology and Science ISTS, 2-9 June 2013. Nagoya, Japan.

3. Barral, S., Kurzyna, J., Remírez, E., Martín, R., Ortiz, P., Alonso, J., Bottinelli, S., Mabillard, Y., Zaldívar, A., Rangsten, P., \& Koppel, C. R. (2013). Development status of an open capillary pulsed plasma thruster

\section{Appendix}

The derivation of the formula for the impulse bit of Eq. (I)

The equation of the dynamics along the $x$ direction of the stand (equation for harmonic oscillator) is:

$$
m \ddot{x}+d \ddot{x}+k x=F(t)
$$

where $m$ is the total moving mass, $d$ is the damping coefficient and $k$ is the stiffness of the guiding device. This can be also presented as:

$$
\ddot{x}+2 \gamma \omega \ddot{x}+\omega^{2} x=\frac{F(t)}{m}
$$

where $\omega=\sqrt{\frac{k}{m}}$ and $\gamma=\frac{d}{2 m \omega}$. with non-volatile liquid propellant. In Proceedings of the 33rd International Electric Propulsion Conference, 6-10 October 2013 (paper IEPC-2013-291). Worthington, OH: The Electric Rocket Propulsion Society.

4. Koizumi, H., Kakami, A., Furuta, Y., Komurasaki, K., \& Arakawa, Y. (2003). Liquid propellant pulsed plasma thruster. In Proceedings of 28th International Electric Propulsion Conference, Toulouse, France (no. 2003-87). Worthington, OH: The Electric Rocket Propulsion Society.

5. Koizumi, H., Kawazoe, Y., Komurasaki, K., \& Arakawa, Y. (2005). Performance improvement of a liquid propellant pulsed plasma thruster. In Proceedings of 29th International Electric Propulsion Conference, Princeton, NJ (no. 2005-69). Worthington, OH: The Electric Rocket Propulsion Society.

6. Scharlemann, C. (2003). Investigation of thrust mechanism in a water fed pulsed plasma thruster. $\mathrm{Ph}$. D. dissertation, The Ohio State University.

7. Krejci, D., Seifert, B., \& Scharleman, C. (2013). Endurance testing of pulsed plasma thruster for nanosatellites. Acta Astronomica, 91, 187-193.

8. Haag, T. (1997). Thrust stand for pulsed plasma thrusters. Rev. Sci. Instrum., 68, 2060-2067.
The solution is:

$$
x(t)=\frac{A}{\sqrt{1-\gamma}} e^{-\gamma \omega t} \sin \left(\omega \sqrt{1-\gamma^{2} t}\right)
$$

and the specific arrangement for $d=0$ and concurrently $\gamma=0$ (device with no damping) takes the following form of foregoing equation:

$$
x(t)=A \sin (\omega t)
$$

and

where $I$ is the expected impulse bit [8]. 